\title{
Partitioning 3-edge-colored complete equi-bipartite graphs by monochromatic trees under a color degree condition*
}

\author{
Xueliang Li and Fengxia Liu \\ Center for Combinatorics and LPMC-TJKLC, \\ Nankai University, Tianjin 300071, P.R. China \\ lxl@nankai.edu.cn, xjulfx@163.com \\ Submitted: Jan 2, 2008; Accepted: Oct 5, 2008; Published: Oct 20, 2008 \\ Mathematics Subject Classifications: 05C05, 05C15, 05C70, 05C35
}

\begin{abstract}
The monochromatic tree partition number of an $r$-edge-colored graph $G$, denoted by $t_{r}(G)$, is the minimum integer $k$ such that whenever the edges of $G$ are colored with $r$ colors, the vertices of $G$ can be covered by at most $k$ vertex-disjoint monochromatic trees. In general, to determine this number is very difficult. For 2edge-colored complete multipartite graph, Kaneko, Kano, and Suzuki gave the exact value of $t_{2}\left(K\left(n_{1}, n_{2}, \cdots, n_{k}\right)\right)$. In this paper, we prove that if $n \geq 3$, and $K(n, n)$ is 3-edge-colored such that every vertex has color degree 3 , then $t_{3}(K(n, n))=3$.
\end{abstract}

Keywords: monochromatic tree, tree partition number, complete bipartite graph, 3-edge-colored, color degree

\section{Introduction}

The monochromatic tree partition number, or simply tree partition number of an $r$ edge-colored graph $G$, denoted by $t_{r}(G)$, which was introduced by Erdős, Gyárfás and Pyber [1], is the minimum integer $k$ such that whenever the edges of $G$ are colored with $r$ colors, the vertices of $G$ can be covered by at most $k$ vertex-disjoint monochromatic trees. Erdős, Gyárfás and Pyber [1] conjectured that the tree partition number of an $r$-edge-colored complete graph is $r-1$. Moreover, they proved that the conjecture is true for $r=3$. For the case $r=2$, it is equivalent to the fact that for any graph $G$, either $G$ or its complement is connected, an old remark of Erdös and Rado.

*Supported by NSFC, PCSIRT and the "973" program. 
For infinite complete graph, Hajnal [2] proved that the tree partition number for an $r$-edge-colored infinite complete graph is at most $r$. For finite complete graph, Haxell and Kohayakawa [3] proved that any $r$-edge-colored complete graph $K_{n}$ contains at most $r$ monochromatic trees, all of different colors, whose vertex sets partition the vertex set of $K_{n}$, provided $n \geq 3 r^{4} r !(1-1 / r)^{3(1-r)} \log r$. In general, to determine the exact value of $t_{r}(G)$ is very difficult.

In this paper we consider the tree partition number of complete bipartite graphs. Notice that isolated vertices are also considered as monochromatic trees. For any $m \geq$ $n \geq 1$, let $K(A, B)=K(m, n)$ denote the complete bipartite graph with partite sets $A$ and $B$, where $|A|=m,|B|=n$. Haxell and Kohayakawa [3] proved that the tree partition number for an $r$-edge-colored complete bipartite graph $K(n, n)$ is at most $2 r$, provided $n$ is sufficiently large. For 2-edge-colored complete multipartite graph $K\left(n_{1}, n_{2}, \cdots, n_{k}\right)$, Kaneko, Kano, and Suzuki [5] proved the following result: Let $n_{1}, n_{2}, \cdots n_{k}(k \geq 2)$ be integers such that $1 \leq n_{1} \leq n_{2} \leq \cdots \leq n_{k}$, and let $n=n_{1}+n_{2}+\cdots+n_{k-1}$ and $m=n_{k}$. Then $t_{2}\left(K\left(n_{1}, n_{2}, \cdots, n_{k}\right)\right)=\left\lfloor\frac{m-2}{2^{n}}\right\rfloor+2$. In particular, $t_{2}(K(m, n))=\left\lfloor\frac{m-2}{2^{n}}\right\rfloor+2$, where $1 \leq n \leq m$. Later in [4], Jin et al gave a polynomial-time algorithm to partition a 2-edgecolored complete multipartite graph into monochromatic trees. For a general survey on monochromatic subgraph partitions, we refer the reader to [6].

In the present paper, we show that if $n \geq 3$ and $K(n, n)$ is 3-edge-colored such that every vertex has color degree 3 , then $t_{3}(K(n, n))=3$, where the color degree of a vertex $v$ is the number of colors of edges incident with $v$.

\section{Preliminaries}

In this section, we will give some notations and results on 2-edge-colored complete bipartite graphs. Although the result on the partition number for 2-edge-colored complete bipartite graphs was obtained by Kaneko, Kano and Suzuki in [5], and a polynomial-time algorithm to get an optimal partition was obtained by Jin et al in [4], in the following we will distinguish several cases, and for each of which we will give the exact monochromatic trees to partition the vertex set of a 2-edge-colored complete bipartite graph. This gives not only the partition number for each case, but more importantly, the clear structural description for the partition, which will plays a key role for obtaining an optimal partition in the 3-edge-colored case.

We first introduce two types of graphs. Let $G=K(A, B)$ be a 2-edge-colored complete bipartite graph, and all the edges are colored with blue or green. If the partite sets $A$ and $B$ have partitions $A=A_{1} \cup A_{2}$ and $B=B_{1} \cup B_{2}$ with $A_{i} \neq \emptyset$ and $B_{i} \neq \emptyset$ such that $K\left(A_{1}, B_{1}\right)$ and $K\left(A_{2}, B_{2}\right)$ are complete bipartite graphs colored with blue, $K\left(A_{1}, B_{2}\right)$ and $K\left(A_{2}, B_{1}\right)$ are complete bipartite graphs colored with green, then we call $K(A, B)$ an $M$-type graph. An $S$-type graph is the graph satisfying blue $(G) \neq \emptyset$ and $\operatorname{green}(G) \neq \emptyset$, where blue $(G)=\{u \mid$ all the edges incident with $u$ are blue $\}$, green $(G)=\{u \mid$ all the edges incident with $u$ are green $\}$. Clearly, both blue $(G)$ and green $(G)$ must be contained in a same partite set $A$ or $B$ of $G$. If $G=K(A, B)$ is an $S$-type graph or an $M$-type graph, then we simply denote it by $G \in \mathcal{S}$ or $G \in \mathcal{M}$. 
Assume that $G=K(A, B)$ is an $S$-type graph. If blue $(G) \cup \operatorname{green}(G) \subseteq A$, then we denote $A_{b}=\{u \in A \mid$ all the edges incident with $u$ are blue $\}, A_{g}=\{u \in A \mid$ all the edges incident with $u$ are green $\}$, and $A_{2}=A-A_{b} \cup A_{g}=\{u \in A \mid$ the color degree of $u$ is 2$\}$. Hence $K\left(A_{b} \cup A_{2}, B\right)$ and $K\left(A_{g} \cup A_{2}, B\right)$ have a blue and green spanning tree, respectively. If blue $(G) \cup \operatorname{green}(G) \subseteq B$, then $B_{b}, B_{g}$ and $B_{2}$ defined analogously and have a similar property.

Lemma 1 The 2-edge-colored complete bipartite graph $K(m, n)$ has a monochromatic spanning tree if and only if $K(m, n) \notin \mathcal{S}$ and $K(m, n) \notin \mathcal{M}$.

Proof. The necessity is obviously. Now we prove the sufficiency.

Assume that $K(m, n)=K(A, B)$ has a vertex $x$ such that all the edges incident with $x$ have the same color. By symmetry, we may assume that the color is blue and $x \in A$. Since $K(m, n) \notin \mathcal{S}$, for every vertex $u$ of $A$, there exists a blue edge incident with $u$. Hence $K(m, n)$ has a blue spanning tree.

We may assume therefore that for any vertex $x$ of $K(m, n)$, at least one blue edge and one green edge are incident with it. Let $H$ be a subgraph of $K(m, n)$ induced by the green edges of $K(m, n)$, and so $H$ is a spanning subgraph. If $H$ is connected, then $H$ contains a green spanning tree of $K(m, n)$, and the lemma follows. Thus, we may assume that $H$ is not connected. Suppose $S$ is a connected component of $H$, and $S \cap A=A_{1}, S \cap B=B_{1}$. Since $S$ is not a spanning subgraph of $K(m, n)$, it follows that $A_{1} \neq A$ and $B_{1} \neq B$. Then $K\left(A_{1}, B-B_{1}\right)$ and $K\left(A-A_{1}, B_{1}\right)$ are both blue complete bipartite graphs. Since $K(m, n) \notin \mathcal{M}$, we have that at least one of $K\left(A_{1}, B_{1}\right)$ and $K\left(A-A_{1}, B-B_{1}\right)$ is not green bipartite graph, and so $K\left(A_{1}, B_{1}\right)$ and $K\left(A-A_{1}, B-B_{1}\right)$ have blue edges. Therefore, $K(m, n)$ has a blue spanning tree.

Lemma 1 implies that if the 2-edge-colored complete bipartite graph $K(m, n)$ does not have a monochromatic spanning tree, then $K(m, n) \in \mathcal{S}$ or $K(m, n) \in \mathcal{M}$.

Lemma 2 Let $K(A, B)$ be a 2-edge-colored complete bipartite graph. If $K(A, B) \in \mathcal{M}$, then the vertices of $K(A, B)$ can be covered by two vertex-disjoint monochromatic trees with the same color.

Proof. Since $K(A, B) \in \mathcal{M}$, we have partitions $A=A_{1} \cup A_{2}$ and $B=B_{1} \cup B_{2}$ such that $K\left(A_{1}, B_{1}\right)$ and $K\left(A_{2}, B_{2}\right)$ are blue complete bipartite graphs, $K\left(A_{1}, B_{2}\right)$ and $K\left(A_{2}, B_{1}\right)$ are green complete bipartite graphs. That is, the vertices of $K(A, B)$ can be covered by two vertex-disjoint blue trees or two green trees.

Assume that $G=K(A, B)$ is an $S$-type graph and blue $(G) \cup \operatorname{green}(G) \subseteq A$. If $A=A_{b} \cup A_{2} \cup A_{g}$ and $B$ satisfies $|B|=1,\left|A_{b}\right| \geq 2$, and $\left|A_{g}\right| \geq 2$, then $K(A, B)$ is call an $S_{1}^{*}$-type graph. If blue $(G) \cup \operatorname{green}(G) \subseteq B$, then an $S_{1}^{*}$-type $K(A, B)$ is defined analogously. Notice that if $K(A, B)$ is call an $S_{1}^{*}$-type graph with $|B|=1$, then $A_{2}=\emptyset$.

Let $K(A, B)$ be an $S$-type graph, and $\operatorname{blue}(G) \cup \operatorname{green}(G) \subseteq A$. Then for partition $B=B_{i} \cup \overline{B_{i}}$, we define

$b\left(B_{i}\right)=\left\{x \in A_{2} \mid K\left(x, B_{i}\right)\right.$ is a blue star, and $K\left(x, \overline{B_{i}}\right)$ is a green star $\}$,

$b\left(\overline{B_{i}}\right)=\left\{x \in A_{2} \mid K\left(x, B_{i}\right)\right.$ is a green star, and $K\left(x, \overline{B_{i}}\right)$ is a blue star $\}$. 
If for every partition $B=B_{i} \cup \overline{B_{i}}$, it follows that $b\left(B_{i}\right) \neq \emptyset, b\left(\overline{B_{i}}\right) \neq \emptyset,\left|A_{b}\right| \geq 2$, $\left|A_{g}\right| \geq 2$, and $|B| \geq 2$, then we call $K(A, B)$ an $S_{1}^{\prime}$-type graph. If blue $(G) \cup \operatorname{green}(G) \subseteq B$, then $b\left(A_{i}\right), b\left(\overline{A_{i}}\right)$, and $S_{1}^{\prime}$-type graph $K(A, B)$ defined analogously.

In the following, the $S_{1}^{*}$-type graphs and the $S_{1}^{\prime}$-type graphs are denoted by $S_{1}$-type graph. The $S$-type graphs other than the $S_{1}$-type graphs are denoted by $S_{2}$-type graph.

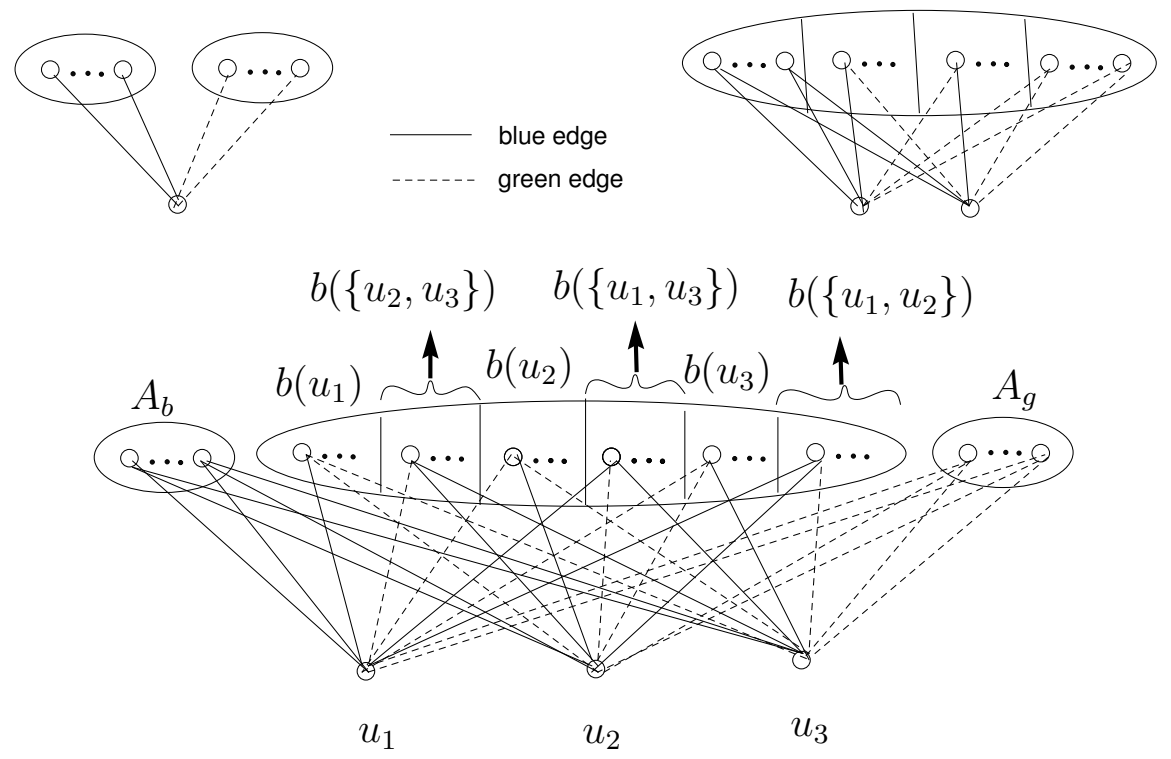

Figure 1: $S_{1}$-type graphs.

Let $K(A, B) \in \mathcal{S}$, blue $(G) \cup \operatorname{green}(G) \subseteq A$, and denote $A=A_{b} \cup A_{2} \cup A_{g}$. If $K(A, B) \in \mathcal{S}_{1}^{*}$, then $A_{2}=\emptyset$, and $|A| \geq 4=2^{|B|}+2$. If $K(A, B) \in \mathcal{S}_{1}^{\prime}$, then $A_{2}=$ $\cup_{B=B_{i} \cup \overline{B_{i}}}\left[b\left(B_{i}\right) \cup b\left(\overline{B_{i}}\right)\right]$, here the union is over all nonempty partitions of $B$, and for any $i, b\left(B_{i}\right) \neq \emptyset$ and $b\left(\overline{B_{i}}\right) \neq \emptyset$. Hence, $\left|A_{2}\right| \geq 2^{|B|}-2$, and so $|A| \geq 2^{|B|}+2$. Thus, if $K(A, B) \in \mathcal{S}_{1}$, then either $|A| \geq 2^{|B|}+2$ or $|B| \geq 2^{|A|}+2$ holds. If $K(A, B) \in \mathcal{S}_{2}$, and $\operatorname{blue}(G) \cup \operatorname{green}(G) \subseteq A$, then either $\min \left\{\left|A_{b}\right|,\left|A_{g}\right|\right\}=1$, or there exists a partition $B=B_{i} \cup \overline{B_{i}}$ such that $b\left(B_{i}\right)=\emptyset$ or $b\left(\overline{B_{i}}\right)=\emptyset$.

Lemma 3 Let $K(A, B)$ be a 2-edge-colored complete bipartite graph. If $K(A, B) \in \mathcal{S}_{2}$, then the vertices of $K(A, B)$ can be covered by either an isolated vertex and a monochromatic tree or two vertex-disjoint monochromatic trees with different colors. Furthermore, except the case $\min \{|\operatorname{blue}(G)|,|\operatorname{green}(G)|\}=1$, the vertices of $K(A, B)$ always can be covered by two vertex-disjoint monochromatic trees colored with different colors.

Proof. Without loss of generality, suppose blue $(G) \cup \operatorname{green}(G) \subseteq A$, and denote $A=$ $A_{b} \cup A_{2} \cup A_{g}$.

Case 1. $\min \left\{\left|A_{b}\right|,\left|A_{g}\right|\right\}=1$. 
Since $K(A, B) \in \mathcal{S}, K\left(A_{b} \cup A_{2}, B\right)$ and $K\left(A_{g} \cup A_{2}, B\right)$ have a monochromatic spanning tree, respectively. Then the vertices of $K(A, B)$ can be covered by an isolated vertex and a monochromatic tree.

Case 2. There exists a partition $B=B_{i} \cup \overline{B_{i}}$ such that $b\left(B_{i}\right)=\emptyset$ or $b\left(\overline{B_{i}}\right)=\emptyset$.

Without loss of generality, suppose $b\left(B_{i}\right)=\emptyset$. Let $A_{21}=\left\{x \in A_{2} \mid K\left(x, \overline{B_{i}}\right)\right.$ have at least one blue edge $\}$, and $A_{22}=A_{2}-A_{21}$. Since $b\left(B_{i}\right)=\emptyset$, every vertex of $A_{22}$ has green edges to $B_{i}$. Then $K\left(A_{b} \cup A_{21}, \overline{B_{i}}\right)$ has a blue spanning tree, and $K\left(A_{g} \cup A_{22}, B_{i}\right)$ has a green spanning tree. Thus, the vertices of $K(A, B)$ can be partitioned by a blue tree and a green tree.

Lemma 4 Let $K(A, B)$ be a 2-edge-colored complete bipartite graph. Then $K(A, B) \in \mathcal{S}_{1}$ if and only if $K(A, B)$ cannot be covered by two vertex-disjoint monochromatic trees.

Proof. We first consider the necessity. Without loss of generality, suppose blue $(G) \cup$ $\operatorname{green}(G) \subseteq A$, and denote $A=A_{b} \cup A_{2} \cup A_{g}$. If $K(A, B) \in \mathcal{S}_{1}^{*}$, then $A_{2}=\emptyset,|B|=1$, and so the vertices of $K(A, B)$ can be covered by at least $\min \left\{\left|A_{b}\right|+1,\left|A_{g}\right|+1\right\} \geq 3$ vertex-disjoint monochromatic trees. For the case $K(A, B) \in \mathcal{S}_{1}^{\prime}$, if all the vertices of $B$ are in one monochromatic tree, then the vertices of $K(A, B)$ can be covered by at least $\min \left\{\left|A_{b}\right|+1,\left|A_{g}\right|+1\right\} \geq 3$ vertex-disjoint monochromatic trees. If all the vertices of $B$ are in two monochromatic trees, since for any partition $B=B=B_{i} \cup \overline{B_{i}}$, we have $b\left(B_{i}\right) \neq \emptyset$ and $b\left(\overline{B_{i}}\right) \neq \emptyset$. So, the vertices of $K(A, B)$ can be covered by at least $\min _{i}\left\{\min _{B=B_{i} \cup \overline{B_{i}}}\left\{\left|b\left(B_{i}\right)\right|,\left|b\left(\overline{B_{i}}\right)\right|\right\}+2\right\} \geq 3$ vertex-disjoint monochromatic trees. If all the vertices of $B$ are in at least three monochromatic trees, then the vertices of $K(A, B)$ can be covered by at least three vertex-disjoint monochromatic trees. In all cases, the vertices of $K(A, B)$ can be covered by at least three vertex-disjoint monochromatic trees.

Now, we prove the sufficiency. If $K(A, B) \notin \mathcal{S}_{1}$, then by the above lemmas, the vertices of $K(A, B)$ can be covered by at most two vertex-disjoint monochromatic trees, a contradiction.

From the above four lemmas, we have

Corollary 5 If $K(A, B)$ is a 2-edge-colored complete bipartite graph, then it has one of the following four structures:

(1) $K(A, B)$ has a monochromatic spanning tree.

(2) $K(A, B) \in \mathcal{M}$.

(3) $K(A, B) \in \mathcal{S}_{2}$.

(4) $K(A, B) \in \mathcal{S}_{1}$.

If $K(A, B)$ satisfies (2) or (3) of Corollary 5 , then by Lemmas 2 and 3 , the vertices of $K(A, B)$ can be covered by at most two vertex-disjoint monochromatic trees. If $K(A, B)$ satisfies (4) of Corollary 5, then from the proof of Lemma 4, the vertices of $K(A, B)$ can be covered by $\min \left\{\left|A_{b}\right|+1,\left|A_{g}\right|+1, \min _{i}\left|b\left(B_{i}\right)\right|+2, \min _{i}\left|b\left(\overline{B_{i}}\right)\right|+2\right\}$ vertex-disjoint monochromatic trees. Notice that $\min \left\{\left|A_{b}\right|+1,\left|A_{g}\right|+1, \min _{i}\left|b\left(B_{i}\right)\right|+2, \min _{i}\left|b\left(\overline{B_{i}}\right)\right|+2\right\} \leq$ $\left\lfloor\frac{m-2}{2^{n}}\right\rfloor+2$, and the equality holds for some graphs. So, the vertices of $K(A, B)$ can be covered by at most $\left\lfloor\frac{m-2}{2^{n}}\right\rfloor+2$ vertex-disjoint monochromatic trees, and there exists an edge 
coloring such that the vertices of $K(A, B)$ are covered by exactly $\left\lfloor\frac{m-2}{2^{n}}\right\rfloor+2$ vertex-disjoint monochromatic trees. Thus, $t_{2}(K(m, n))=\left\lfloor\frac{m-2}{2^{n}}\right\rfloor+2$.

Let $K(A, B)$ be a 3-edge-colored complete bipartite graph, all the edges of $K(A, B)$ are red, blue or green. Given a monochromatic tree partition of $K(A, B)$, the following cases can be distinguished:

Case A: $A$ does not contain isolated vertices, and all the vertices of $A$ are in blue trees and green trees.

Case B: $A$ does not contain isolated vertices, and there exist some vertices of $A$ that are in a red tree.

Case C: $A$ contains some isolated vertices, and all the other vertices of $A$ are in blue trees and green trees.

Case D: $A$ contains some isolated vertices, and there exist some vertices of $A$ that are in a red tree.

Lemma 6 Let $K(A, B)$ be a 3-edge-colored complete bipartite graph. If $|A| \leq|B|$, then there exists a monochromatic tree partition belonging to Case $A$ or Case B. If $|A|>|B|$, then there exists a monochromatic tree partition belonging to Case A, Case B or Case C.

Proof. Let MTP be an extremal monochromatic tree partition of $K(A, B)$ satisfying the following three conditions:

(c1) the number of vertices of $A$ that are contained in blue trees and green trees is maximum;

(c2) subject to (c1), the number of vertices of $B$ that are contained in blue trees and green trees is minimum;

(c3) subject to (c1) and (c2), the number of monochromatic trees is minimum.

In the following, we will prove that the MTP is a required monochromatic tree partition of this lemma.

We use $A_{b g}$ to denote the vertices of $A$ that are contained in blue trees and green trees in the MTP, and denote $A_{0}=A-A_{b g} . B_{b g}$ and $B_{0}$ are defined similarly. If $A_{0}=\emptyset$, then the MTP belongs to Case A. In the following we consider the case $A_{0} \neq \emptyset$. Since the MTP satisfies (c2), it follows that $\left|A_{b g}\right| \geq\left|B_{b g}\right|$. If $|A| \leq|B|$ and $A_{0} \neq \emptyset$, then $B_{0} \neq \emptyset$. But for $|A|>|B|$, both of $B_{0}=\emptyset$ and $B_{0} \neq \emptyset$ may occur. If $B_{0} \neq \emptyset$, then all the edges of $K\left(A_{0}, B_{0}\right)$ are red, otherwise contradicts to (c1). Thus, the MTP belongs to Case B. If $B_{0}=\emptyset$, then the MTP belongs to Case C. Thus, the lemma holds.

\section{Main result}

Theorem 7 If $n \geq 3$, and $K(n, n)$ is 3-edge-colored such that every vertex has color degree 3 , then $t_{3}(k(n, n))=3$.

Proof. Assume that all the edges of $K(n, n)(=K(A, B))$ are blue, green, or red. The vertices of the graph in Figure 2 are covered by at least three vertex-disjoint monochromatic trees. Then, $t_{3}(k(n, n)) \geq 3$. 


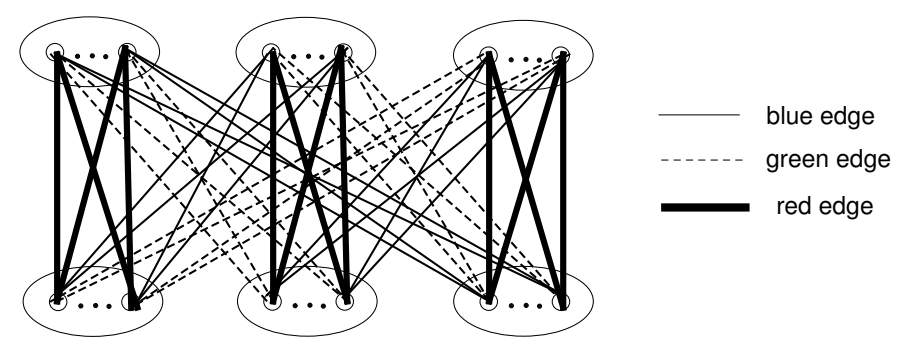

Figure 2. Graph satisfying that the vertices can be partitioned into at least 3 monochromatic trees.

In the following, we prove $t_{3}(k(n, n)) \leq 3$. Suppose $R$ is the monochromatic connected component of $K(A, B)$ with the maximum number of vertices, without loss of generality, suppose $R$ is red. Denote $R=R_{1} \cup R_{2}, R_{1}=R \cap A$, and $R_{2}=R \cap B$.

If $R_{1}=A$, since the color degree of every vertex is 3 , we have $R_{2}=B$, then $K(A, B)$ has a red spanning tree.

We may assume therefore that $R_{1} \neq A$ and $R_{2} \neq B$. Denote $C=A-R_{1}$ and $D=B-R_{2}$. Clearly, all the edges of $K\left(R_{1}, D\right)$ and $K\left(R_{2}, C\right)$ are blue or green.

If the vertices of $K(C, D)$ can be covered by at most two vertex-disjoint monochromatic trees, then the vertices of $K(A, B)$ can be covered by at most three vertex-disjoint monochromatic trees. Thus, in the following, we assume that the vertices of $K(C, D)$ can be covered by at least three vertex-disjoint monochromatic trees.

Claim 1. Every vertex in $K(C, D)$ has at least one red edge incident with it, and there are at least one green edge and one blue edge in $K(C, D)$.

Proof. Since every vertex has color degree 3 , and $K\left(R_{1}, D\right)$ and $K\left(R_{2}, C\right)$ are 2-edgecolored graphs colored with blue and green, it is obvious that every vertex in $K(C, D)$ has at least one red edge incident with it. Since the vertices of $K(C, D)$ can be covered by at least three vertex-disjoint monochromatic trees, the edges of $K(C, D)$ must be colored by at least two colors. Without loss of generality, we assume $K(C, D)$ does not have green edges, that is, $K(C, D)$ is a 2-edge-colored graph colored with blue and red. By Lemma 4 we have $K(C, D) \in S_{1}$. Then, $K(C, D)$ has a vertex such that all the edges incident with it are blue, which contradicts the fact that every vertex in $K(C, D)$ has at least one red edge incident with it. Thus, $K(C, D)$ has green edges.

Claim 2. $|C| \geq 3$ and $|D| \geq 3$.

Proof. Suppose $|C| \leq 2$. By Claim 1 every vertex in $K(C, D)$ has at least one red edge incident with it, then the vertices of $K(C, D)$ can be covered by two vertex-disjoint red stars or a red spanning tree, which contradicts the assumption that the vertices of $K(C, D)$ can be covered by at least three vertex-disjoint monochromatic trees.

Since $K\left(R_{1}, D\right)$ and $K\left(R_{2}, C\right)$ are 2-edge-colored graphs colored with blue and green, by Corollary 5 we consider the following eight cases:

Case 1. Both $K\left(R_{1}, D\right)$ and $K\left(R_{2}, C\right)$ have monochromatic spanning trees. 
Case 2. One of $K\left(R_{1}, D\right)$ and $K\left(R_{2}, C\right)$ has a monochromatic tree, the other is an $M$-type graph or an $S_{2}$-type graph.

Case 3. One of $K\left(R_{1}, D\right)$ and $K\left(R_{2}, C\right)$ has a monochromatic tree, the other is an $S_{1}$-type graph.

Case 4. $K\left(R_{1}, D\right) \in \mathcal{M}$ and $K\left(R_{2}, C\right) \in \mathcal{M}$.

Case 5. One of $K\left(R_{1}, D\right)$ and $K\left(R_{2}, C\right)$ is an $M$-type graph, the other is an $S$-type graph.

Case 6. $K\left(R_{1}, D\right) \in \mathcal{S}_{2}$ and $K\left(R_{2}, C\right) \in \mathcal{S}_{2}$.

Case 7. $K\left(R_{1}, D\right) \in \mathcal{S}_{1}$ and $K\left(R_{2}, C\right) \in \mathcal{S}_{1}$.

Case 8. One of $K\left(R_{1}, D\right)$ and $K\left(R_{2}, C\right)$ is an $S_{1}$-type graph, the other is an $S_{2}$-type graph.

In the following, we prove that for every above case, the vertices of $K(A, B)$ can be covered by at most three vertex-disjoint monochromatic trees.

Clearly, in Case 1 the vertices of $K(A, B)$ can be covered by at most two vertex-disjoint monochromatic trees. In Case 2 , the vertices of $K(A, B)$ can be covered by at most three vertex-disjoint monochromatic trees.

For Case 3, without loss of generality, suppose $K\left(R_{1}, D\right)$ has a green spanning tree, and $K\left(R_{2}, C\right) \in \mathcal{S}_{1}$. Since $K\left(R_{2}, C\right) \in \mathcal{S}_{1}$, we have $|C| \geq 2^{\left|R_{2}\right|}+2$ or $\left|R_{2}\right| \geq 2^{|C|}+2$. Since $R$ is the maximum monochromatic component, and $K\left(R_{1}, D\right)$ has a green spanning tree, we have $|D| \leq\left|R_{2}\right|$. If $|C| \geq 2^{\left|R_{2}\right|}+2>2\left|R_{2}\right|$, then $|C|>2\left|R_{2}\right|=\left|R_{2}\right|+\left|R_{2}\right| \geq$ $\left|R_{2}\right|+|D|$, contradicting to $\left|R_{1}\right|+|C|=\left|R_{2}\right|+|D|=n$. If $\left|R_{2}\right| \geq 2^{|C|}+2$, that is blue $\left(K\left(R_{2}, C\right)\right) \cup \operatorname{green}\left(K\left(R_{2}, C\right)\right) \subseteq R_{2}$, then denote $R_{2 b}=\left\{u \in R_{2} \mid\right.$ all the edges incident with $u$ are blue in $\left.K\left(R_{2}, C\right)\right\}, R_{2 g}=\left\{u \in R_{2} \mid\right.$ all the edges incident with $u$ are green in $\left.K\left(R_{2}, C\right)\right\}$, and $R_{22}=R_{2}-R_{2 b} \cup R_{2 g}=\left\{u \in R_{2} \mid\right.$ the color degree of $u$ is 2 in $\left.K\left(R_{2}, C\right)\right\}$. Since every vertex has color degree 3 , in $K\left(R_{1}, R_{2 b}\right)$, every vertex in $R_{2 b}$ has at least one green edge incident with it, and so $K\left(R_{1}, R_{2 b} \cup D\right)$ has a green spanning tree. Obviously, $K\left(C, R_{22} \cup R_{2 g}\right)$ has a green spanning tree. Moreover, by Claim $1, K(C, D)$ has at least one green edge. Hence, $K(A, B)$ has a green spanning tree, which contradicts our assumption that $R$ is the maximum monochromatic component. Thus, this case does not occur.

For Case 4, we have $K\left(R_{1}, D\right) \in \mathcal{M}$ and $K\left(R_{2}, C\right) \in \mathcal{M}$. By Lemma 2 the vertices of $K\left(R_{1}, D\right)$ and $K\left(R_{2}, C\right)$ can be covered by two vertex-disjoint green trees, respectively. By Claim $1 K(C, D)$ has at least one green edge. Thus, the vertices of $K(A, B)$ can be covered by at most three vertex-disjoint green trees.

For Case 5, without loss of generality, suppose $K\left(R_{1}, D\right) \in \mathcal{M}, K\left(R_{2}, C\right) \in \mathcal{S}$. Since $K\left(R_{2}, C\right) \in \mathcal{S}$, we can denote $R_{2}=R_{2 b} \cup R_{22} \cup R_{2 g}$ or $C=C_{b} \cup C_{2} \cup C_{g}$. If $R_{2}=$ $R_{2 b} \cup R_{22} \cup R_{2 g}$, then $K\left(C, R_{22} \cup R_{2 g}\right)$ has a green spanning tree. Since every vertex has color degree 3 , in $K\left(R_{1}, R_{2 b}\right)$ every vertex in $R_{2 b}$ is incident with at least one green edge. By Lemma 2 the vertices of $K\left(R_{1}, D\right)$ can be covered by two vertex-disjoint green trees. Then, the vertices of $K\left(R_{1}, R_{2 b} \cup D\right)$ can be covered by at most two vertex-disjoint green trees. Moreover, $K(C, D)$ has at least one green edge. Thus, the vertices of $K(A, B)$ can 
be covered by at most two vertex-disjoint green trees. If $C=C_{b} \cup C_{2} \cup C_{g}$, by a similar argument, the vertices of $K\left(R_{1} \cup C_{b}, D\right)$ can be covered by at most two vertex-disjoint green trees, and $K\left(C_{2} \cup C_{g}, R_{2}\right)$ has a green spanning tree. Thus, the vertices of $K(A, B)$ can be covered by at most three vertex-disjoint green trees.

For Case 6, we have $K\left(R_{1}, D\right) \in \mathcal{S}_{2}$ and $K\left(R_{2}, C\right) \in \mathcal{S}_{2}$. Since $K\left(R_{1}, D\right) \in \mathcal{S}_{2}$, we can denote $R_{1}=R_{1 b} \cup R_{12} \cup R_{1 g}$ or $D=D_{b} \cup D_{2} \cup D_{g}$. Similarly, we have $R_{2}=R_{2 b} \cup R_{22} \cup R_{2 g}$ or $C=C_{b} \cup C_{2} \cup C_{g}$.

Subcase 6.1. $R_{1}=R_{1 b} \cup R_{12} \cup R_{1 g}$ and $C=C_{b} \cup C_{2} \cup C_{g}$.

Since every vertex has color degree 3 , in $K\left(R_{1 b}, R_{2}\right)$ every vertex in $R_{1 b}$ has at least one green edge incident with it. In $K\left(C_{b}, D\right)$ every vertex in $C_{b}$ has at least one green edge incident with it. Then $K\left(R_{1 b} \cup C_{2} \cup C_{g}, R_{2}\right)$ and $K\left(R_{12} \cup R_{1 g} \cup C_{b}, D\right)$ have a green spanning tree, respectively. Thus, the vertices of $K(A, B)$ can be covered by at most two vertex-disjoint green trees.

Subcase 6.2. $R_{2}=R_{2 b} \cup R_{22} \cup R_{2 g}$ and $D=D_{b} \cup D_{2} \cup D_{g}$.

The proof is similar to that of Subcase 6.1.

Subcase 6.3. $R_{1}=R_{1 b} \cup R_{12} \cup R_{1 g}$ and $R_{2}=R_{2 b} \cup R_{22} \cup R_{2 g}$.

Since $K\left(R_{1}, D\right) \in \mathcal{S}_{2}$ and $K\left(R_{2}, C\right) \in \mathcal{S}_{2}$, we can give the following partition of $R_{1}, C, R_{2}$ and $D$, respectively: $R_{1}=R_{1}^{b} \cup R_{1}^{g}, C=C^{b} \cup C^{g}, R_{2}=R_{2}^{b} \cup R_{2}^{g}$, and $D=D^{b} \cup D^{g}$ such that $K\left(R_{1}^{b}, D^{b}\right)$ has a blue spanning tree, $K\left(R_{1}^{g}, D^{g}\right)$ has a green spanning tree, $K\left(C^{b}, R_{2}^{b}\right)$ has a blue spanning tree, and $K\left(C^{g}, R_{2}^{g}\right)$ has a green spanning tree. Obviously, $R_{1 b} \subseteq R_{1}^{b}, R_{2 b} \subseteq R_{2}^{b}, R_{1 g} \subseteq R_{1}^{g}$ and $R_{2 g} \subseteq R_{2}^{g}$. If $K\left(R_{1 b}, R_{2 b}\right)$ has at least one blue edge, then $K\left(R_{1}^{b}, R_{2}^{b}\right)$ has at least one blue edge. Thus, the vertices of $K(A, B)$ can be covered by at most one blue tree and two green trees. We may assume therefore that all the edges of $K\left(R_{1 b}, R_{2 b}\right)$ are red or green. Since $K(C, D)$ has at least one green edge, $K\left(A-R_{1 b}, B-R_{2 b}\right)$ has a green spanning tree. If the vertices of $K\left(R_{1 b}, R_{2 b}\right)$ can be covered by at most two vertex-disjoint monochromatic trees, then the vertices of $K(A, B)$ can be covered by at most three vertex-disjoint monochromatic trees. So, we assume that the vertices of $K\left(R_{1 b}, R_{2 b}\right)$ can be covered by at least three vertex-disjoint monochromatic trees. By Lemma 4 we have $K\left(R_{1 b}, R_{2 b}\right) \in \mathcal{S}_{1}$. Without loss of generality, we assume that $R_{1 b}^{r}$ is the set with maximum number of vertices such that $K\left(R_{1 b}^{r}, R_{2 b}\right)$ is a red complete bipartite graph. Then $K\left(R_{1 b}-R_{1 b}^{r}, R_{2 b}\right)$ has a green spanning tree. Since every vertex has color degree 3 , in $K\left(R_{1 b}^{r}, R_{22} \cup R_{2 g}\right)$ every vertex in $R_{1 b}^{r}$ has at least one green edge incident with it, and so $K\left(R_{1 b}^{r} \cup R_{12} \cup R_{1 g} \cup C, R_{22} \cup R_{2 g} \cup D\right)$ has a green spanning tree. Thus, the vertices of $K(A, B)$ can be covered by two vertex-disjoint green trees.

Subcase 6.4. $C=C_{b} \cup C_{2} \cup C_{g}$ and $D=D_{b} \cup D_{2} \cup D_{g}$.

By the same arguments as in Case 6.3, we have partitions $C=C^{b} \cup C^{g}$ and $D=$ $D^{b} \cup D^{g}$. Clearly, $C_{b} \subseteq C^{b}, C_{g} \subseteq C^{g}, D_{b} \subseteq D^{b}$ and $D_{g} \subseteq D^{g}$. If $K\left(C_{b}, D_{b}\right)$ has at least one blue edge, or $K\left(C_{g}, D_{g}\right)$ has at least one green edge, then the vertices of $K(A, B)$ can be covered by at most three vertex-disjoint monochromatic trees. So, we assume that $K\left(C_{b}, D_{b}\right)$ does not have blue edges, and $K\left(C_{g}, D_{g}\right)$ does not have green edges. Then we 
have the following three subcases.

Subcase 6.4.1. $K\left(C_{b}, D_{b}\right)$ or $K\left(C_{g}, D_{g}\right)$ has a monochromatic spanning tree.

Since each of $K\left(C_{2} \cup C_{g}, R_{2}\right), K\left(D_{2} \cup D_{g}, R_{1}\right), K\left(C_{b} \cup C_{2}, R_{2}\right)$ and $K\left(D_{b} \cup D_{2}, R_{1}\right)$ has a monochromatic spanning tree, the vertices of $K(A, B)$ can be covered by at most three vertex-disjoint monochromatic trees.

Subcase 6.4.2. $K\left(C_{b}, D_{b}\right) \in \mathcal{S}$ or $K\left(C_{g}, D_{g}\right) \in \mathcal{S}$.

By a similar proof to the later part of Case 6.3, we can obtain that the vertices of $K(A, B)$ can be covered by at most three vertex-disjoint monochromatic trees.

Subcase 6.4.3. $K\left(C_{b}, D_{b}\right) \in \mathcal{M}$ and $K\left(C_{g}, D_{g}\right) \in \mathcal{M}$, see Figure 3 .

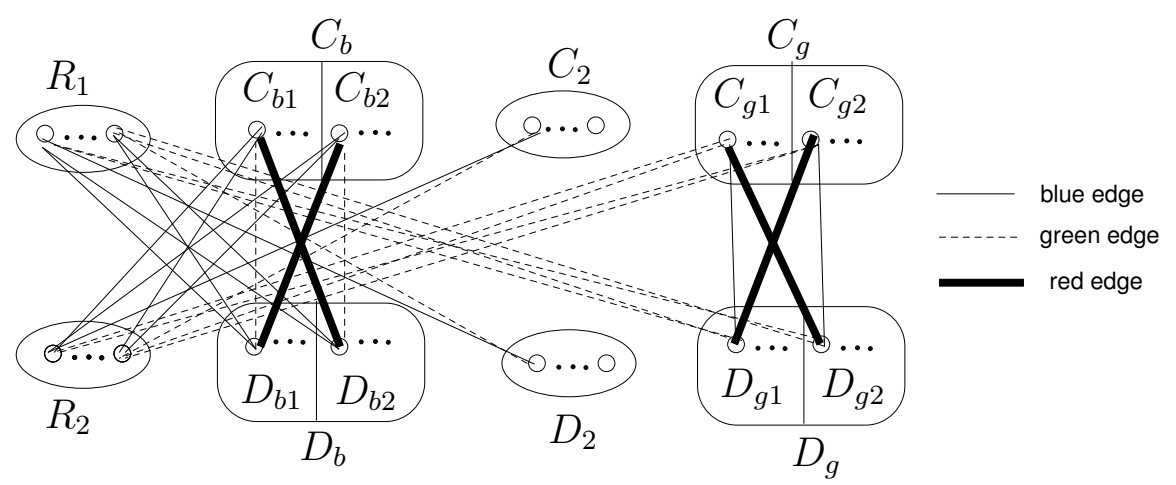

Figure 3: The graph of Subcase 6.4.3.

If all the edges of $K\left(C_{b}, D_{g}\right)$ are red, then $K\left(C_{b 1} \cup C_{g}, D_{g}\right)$ has a red spanning tree. Since $K\left(C_{b 2} \cup C_{2}, R_{2}\right)$ and $K\left(R_{1}, D_{b} \cup D_{2}\right)$ have blue spanning trees, the vertices of $K(A, B)$ can be covered by three vertex-disjoint monochromatic trees. Thus, we may assume that $K\left(C_{b}, D_{g}\right)$ has at least one green edge or at least one blue edge. Without loss of generality, assume $K\left(C_{b}, D_{g 1}\right)$ has at least one blue edge. Then $K\left(C_{b} \cup C_{2} \cup C_{g 1}, R_{2} \cup D_{g 1}\right), K\left(C_{g 2}, D_{g 2}\right)$ and $K\left(R_{1}, D_{b} \cup D_{2}\right)$ has blue spanning trees. Thus, the vertices of $K(A, B)$ can be covered by three vertex-disjoint blue trees.

For Case 7, we have $K\left(R_{1}, D\right) \in \mathcal{S}_{1}$ and $K\left(R_{2}, C\right) \in \mathcal{S}_{1}$. Since $K\left(R_{1}, D\right) \in \mathcal{S}_{1}$, we can denote $R_{1}=R_{1 b} \cup R_{12} \cup R_{1 g}$ or $D=D_{b} \cup D_{2} \cup D_{g}$. Similarly, we have $R_{2}=R_{2 b} \cup R_{22} \cup R_{2 g}$ or $C=C_{b} \cup C_{2} \cup C_{g}$.

Subcase 7.1. $R_{1}=R_{1 b} \cup R_{12} \cup R_{1 g}$ and $C=C_{b} \cup C_{2} \cup C_{g}$.

Since $K\left(R_{1}, D\right) \in \mathcal{S}_{1}$ and $K\left(R_{2}, C\right) \in \mathcal{S}_{1}$, we have $\left|R_{1}\right| \geq 2^{|D|}+2>2|D|$ and $|C| \geq 2^{\left|R_{2}\right|}+2>2\left|R_{2}\right|$, and so $\left|R_{1}\right|+|C|>2\left|R_{2}\right|+2|D|$, contradicting to $\left|R_{1}\right|+|C|=$ $\left|R_{2}\right|+|D|=n$. Thus, this case does not occur.

Subcase 7.2. $R_{2}=R_{2 b} \cup R_{22} \cup R_{2 g}$ and $D=D_{b} \cup D_{2} \cup D_{g}$.

The proof is similarly as Subcase 7.1. 
Subcase 7.3. $C=C_{b} \cup C_{2} \cup C_{g}$ and $D=D_{b} \cup D_{2} \cup D_{g}$.

Clearly, $K\left(C_{b} \cup C_{2}, R_{2}\right)$ and $K\left(C_{2} \cup C_{g}, R_{2}\right)$ have monochromatic spanning trees, and $R$ is the maximum monochromatic tree, then we have $\left|R_{1}\right| \geq \frac{|C|}{2}$. Similarly, $\left|R_{2}\right| \geq \frac{|D|}{2}$. Moreover, by $K\left(R_{1}, D\right) \in \mathcal{S}_{1}$, we have $|D| \geq 2^{\left|R_{1}\right|}+2>2\left|R_{1}\right|$. So, $\left|R_{2}\right| \geq \frac{|D|}{2}>\left|R_{1}\right| \geq \frac{|C|}{2}$, that is, $\left|R_{2}\right|+|D|>\left|R_{1}\right|+|C|$, a contradiction. Thus, this case does not occur.

Subcase 7.4. $R_{1}=R_{1 b} \cup R_{12} \cup R_{1 g}$ and $R_{2}=R_{2 b} \cup R_{22} \cup R_{2 g}$.

We define $R_{1 b}^{(2)}=\left\{u \in R_{1 b} \mid K(A, B)\right.$ contains a green $u v$-path for some $v \in R_{1}-R_{1 b}$ or $\left.v \in R_{2}-R_{2 b}\right\}, R_{1 b}^{(1)}=R_{1 b}-R_{1 b}^{(2)} ; R_{1 g}^{(2)}=\left\{u \in R_{1 g} \mid K(A, B)\right.$ contains a blue $u v$-path for some $v \in R_{1}-R_{1 g}$ or $\left.v \in R_{2}-R_{2 g}\right\}, R_{1 g}^{(1)}=R_{1 g}-R_{1 g}^{(2)} ; R_{2 b}^{(1)}, R_{2 b}^{(2)}, R_{2 g}^{(1)}$ and $R_{2 g}^{(2)}$ are defined similarly.

Clearly, $K\left(R_{1 b}^{(1)}, R_{2}-R_{2 b}^{(1)}\right)$ and $K\left(R_{2 b}^{(1)}, R_{1}-R_{1 b}^{(1)}\right)$ do not have green edges, $K\left(R_{1 g}^{(1)}\right.$, $\left.R_{2}-R_{2 g}^{(1)}\right)$ and $K\left(R_{2 g}^{(1)}, R_{1}-R_{1 g}^{(1)}\right)$ do not have blue edges. By Claim $1, K(C, D)$ has at least one blue edge and one green edge, then $K\left(A-R_{1 b}^{(1)}, B-R_{2 b}^{(1)}\right)$ has a green spanning tree, $K\left(A-R_{1 g}^{(1)}, B-R_{2 g}^{(1)}\right)$ has a blue spanning tree. If the vertices of $K\left(R_{1 b}^{(1)}, R_{2 b}^{(1)}\right)$ or $K\left(R_{1 g}^{(1)}, R_{2 g}^{(1)}\right)$ can be covered by at most two vertex-disjoint monochromatic trees, then the vertices of $K(A, B)$ can be covered by at most three vertex-disjoint monochromatic trees. In the following, we consider the case that the vertices of both $K\left(R_{1 b}^{(1)}, R_{2 b}^{(1)}\right)$ and $K\left(R_{1 g}^{(1)}, R_{2 g}^{(1)}\right)$ can be covered by at least three vertex-disjoint monochromatic trees. We first give several remarks.

Remark 1. $K\left(R_{1 b}^{(1)}, R_{2 g}^{(1)}\right)$ and $K\left(R_{1 g}^{(1)}, R_{2 b}^{(1)}\right)$ are red complete bipartite graphs.

Since every vertex has color degree 3 , we have

Remark 2. Every vertex in $K\left(R_{1 b}^{(1)}, R_{2 b}^{(1)}\right)$ has at least one green edge incident with it, and every vertex in $K\left(R_{1 g}^{(1)}, R_{2 g}^{(1)}\right)$ has at least one blue edge incident with it.

Since $R$ is the maximum monochromatic component, we have

Remark 3. $\left|R_{1 b}^{(1)}\right|+\left|R_{2 b}^{(1)}\right| \geq|C|+|D|$ and $\left|R_{1 g}^{(1)}\right|+\left|R_{2 g}^{(1)}\right| \geq|C|+|D|$.

Remark 4. $\forall i=1,2, j=b, g,\left|R_{i j}^{(1)}\right| \geq 3$.

Proof. Without loss of generality, suppose $\left|R_{1 b}^{(1)}\right| \leq 2$. If $R_{1 b}^{(1)}=\emptyset$, since every vertex has color degree 3 , and $K\left(R_{2 b}^{(1)}, A\right)$ has only blue edges and red edges, we have $R_{2 b}^{(1)}=\emptyset$, which contradicts to the assumption that the vertices of $K\left(R_{1 b}^{(1)}, R_{2 b}^{(1)}\right)$ can be covered by at least three vertex-disjoint monochromatic trees. If $1 \leq\left|R_{1 b}^{(1)}\right| \leq 2$, then the vertices of $K\left(R_{1 b}^{(1)}, R_{2 b}^{(1)}\right)$ can be covered by one green star or at most two vertex-disjoint green trees, a contradiction.

Remark 5. $K\left(R_{1 b}^{(1)}, R_{2 b}^{(1)}\right)$ has at least one red edge and one blue edge, $K\left(R_{1 g}^{(1)}, R_{2 g}^{(1)}\right)$ has at least one red edge and one green edge.

Proof. If $K\left(R_{1 b}^{(1)}, R_{2 b}^{(1)}\right)$ does not have red edges, by Remark $2, K\left(R_{1 b}^{(1)}, R_{2 b}^{(1)}\right)$ does not have any vertex such that all the edges incident with it are blue, and so either $K\left(R_{1 b}^{(1)}, R_{2 b}^{(1)}\right)$ has 
a monochromatic tree, or $K\left(R_{1 b}^{(1)}, R_{2 b}^{(1)}\right) \in \mathcal{M}$, which contradicts to the assumption that the vertices of $K\left(R_{1 b}^{(1)}, R_{2 b}^{(1)}\right)$ can be covered by at least three vertex-disjoint monochromatic trees. For other cases, we can prove them similarly.

Remark 6. In $K\left(R_{1 b}^{(1)}, R_{2 b}^{(1)}\right)$, every blue edge has at least one red edge and one blue edge independent of it, every red edge has at least one red edge and one blue edge independent of it. $K\left(R_{1 g}^{(1)}, R_{2 g}^{(1)}\right)$ have a similar property.

Proof. Let $e=u v$ be a blue edge of $K\left(R_{1 b}^{(1)}, R_{2 b}^{(1)}\right)$. If $K\left(R_{1 b}^{(1)}, R_{2 b}^{(1)}\right)$ does not have red edges independent of $e$, then $K\left(R_{1 b}^{(1)}-u, R_{2 b}^{(1)}-v\right)$ is a 2-edge-colored complete bipartite graph colored with blue and green. If $K\left(R_{1 b}^{(1)}-u, R_{2 b}^{(1)}-v\right)$ has a monochromatic spanning tree, then the vertices of $K\left(R_{1 b}^{(1)}, R_{2 b}^{(1)}\right)$ can be covered by at most two vertex-disjoint monochromatic trees, a contradiction. If $K\left(R_{1 b}^{(1)}-u, R_{2 b}^{(1)}-v\right) \in \mathcal{M}$, then the vertices of $K\left(R_{1 b}^{(1)}-u, R_{2 b}^{(1)}-v\right)$ can be covered by two vertex-disjoint green trees. Since $K\left(R_{1 b}^{(1)}-u, v\right)$ and $K\left(R_{2 b}^{(1)}-v, u\right)$ both have green edges, the vertices of $K\left(R_{1 b}^{(1)}, R_{2 b}^{(1)}\right)$ can be covered by at most two vertex-disjoint green trees, a contradiction. If $K\left(R_{1 b}^{(1)}-u, R_{2 b}^{(1)}-v\right) \in \mathcal{S}$, noticing that $K\left(R_{1 b}^{(1)}-u, v\right)$ and $K\left(R_{2 b}^{(1)}-v, u\right)$ have green edges, then the vertices of $K\left(R_{1 b}^{(1)}, R_{2 b}^{(1)}\right)$ can be covered by a green tree and a green star, a contradiction. Thus, $K\left(R_{1 b}^{(1)}, R_{2 b}^{(1)}\right)$ has red edges independent of $e$. The others can be proved similarly.

Since $|C| \geq 3$ and $|D| \geq 3$, we have $K\left(R_{1}, D\right) \in \mathcal{S}_{1}^{\prime}$ and $K\left(R_{2}, C\right) \in \mathcal{S}_{1}^{\prime}$. Then $R_{12}=\cup_{D=D_{i} \cup \overline{D_{i}}}\left[b\left(D_{i}\right) \cup b\left(\overline{D_{i}}\right)\right]$ and $R_{22}=\cup_{C=C_{i} \cup \overline{C_{i}}}\left[b\left(C_{i}\right) \cup b\left(\overline{C_{i}}\right)\right]$, here the union is over all nonempty partitions of $D$ and $C$, respectively. For any nonempty partitions of $C$ and $D: C=C_{i 1} \cup C_{i 2}, D=D_{i 1} \cup D_{i 2}$, if $\left|b\left(C_{i 1}\right)\right| \geq\left|b\left(C_{i 2}\right)\right|$, then we denote $C_{i 1}=C_{i}, C_{i 2}=\overline{C_{i}}$; if $\left|b\left(D_{i 1}\right)\right| \geq\left|b\left(D_{i 2}\right)\right|$, then we denote $D_{i 1}=D_{i}, D_{i 2}=\overline{D_{i}}$. So, in the following, if we write $C=C_{i} \cup \overline{C_{i}}, D=D_{i} \cup \overline{D_{i}}$, then $\left|b\left(C_{i}\right)\right| \geq\left|b\left(\overline{C_{i}}\right)\right|$ and $\left|b\left(D_{i}\right)\right| \geq\left|b\left(\overline{D_{i}}\right)\right|$.

Subcase 7.4.1. There exist partitions $C=C_{k} \cup \overline{C_{k}}$ and $D=D_{k} \cup \overline{D_{k}}$ such that $\left|b\left(C_{k}\right)\right| \geq\left|b\left(\overline{D_{k}}\right)\right|$ and $\left|b\left(D_{k}\right)\right| \geq\left|b\left(\overline{C_{k}}\right)\right|$.

In this case, $b\left(\overline{C_{k}}\right)$ and $b\left(\overline{D_{k}}\right)$ correspond to the partite set $A$ in Lemma 6 . Then by Lemma $6, K\left(b\left(D_{k}\right), b\left(\overline{C_{k}}\right)\right)$ and $K\left(b\left(C_{k}\right), b\left(\overline{D_{k}}\right)\right)$ have tree partitions satisfying Case A or Case B.

Subcase 7.4.1.1. Both $K\left(b\left(D_{k}\right), b\left(\overline{C_{k}}\right)\right)$ and $K\left(b\left(C_{k}\right), b\left(\overline{D_{k}}\right)\right)$ have tree partitions satisfying Case A.

By Remark $5, K\left(R_{1 b}^{(1)}, R_{2 b}^{(1)}\right)$ has at least one blue edge, $K\left(R_{1 g}^{(1)}, R_{2 g}^{(1)}\right)$ has at least one green edge. Then, $K\left(R_{1 b} \cup C_{k}, R_{2 b} \cup D_{k}\right)$ has a blue spanning tree, and $K\left(R_{1 g} \cup \overline{C_{k}}, R_{2 g} \cup \overline{D_{k}}\right)$ has a green spanning tree. By the definition of $b\left(D_{k}\right)$ and $b\left(C_{k}\right)$, the vertices in $b\left(D_{k}\right)$ and $b\left(C_{k}\right)$ can be connected into the blue tree of $K\left(R_{1 b} \cup C_{k}, R_{2 b} \cup D_{k}\right)$, and they also can be connected into the green tree of $K\left(R_{1 g} \cup \overline{C_{k}}, R_{2 g} \cup \overline{D_{k}}\right)$. Thus, the vertices of $b\left(\overline{C_{k}}\right)$ and $b\left(\overline{D_{k}}\right)$ can be connected into either the blue tree of $K\left(R_{1 b} \cup C_{k}, R_{2 b} \cup D_{k}\right)$ or the green tree of $K\left(R_{1 g} \cup \overline{C_{k}}, R_{2 g} \cup \overline{D_{k}}\right)$ by the vertices in $b\left(D_{k}\right)$ and $b\left(C_{k}\right)$. Moreover, the vertices of $R_{12}-b\left(D_{k}\right)-b\left(\overline{D_{k}}\right)$ have either blue edges to $D_{k}$, or green edges to $\overline{D_{k}}$, the vertices of $R_{22}-b\left(C_{k}\right)-b\left(\overline{C_{k}}\right)$ have either blue edges to $C_{k}$, or green edges to $\overline{C_{k}}$. Thus, the vertices 
of $K(A, B)$ can be covered by a blue tree and a green tree.

Subcase 7.4.1.2. One of $K\left(b\left(D_{k}\right), b\left(\overline{C_{k}}\right)\right)$ and $K\left(b\left(C_{k}\right), b\left(\overline{D_{k}}\right)\right)$ has a tree partition satisfying Case $\mathrm{A}$, the other has a tree partition satisfying Case B.

By a similar argument to that of Subcase 7.4.1.1, the vertices of $K(A, B)$ can be covered by a blue tree, a green tree and a red tree.

Subcase 7.4.1.3. Both $K\left(b\left(D_{k}\right), b\left(\overline{C_{k}}\right)\right)$ and $K\left(b\left(C_{k}\right), b\left(\overline{D_{k}}\right)\right)$ have tree partitions satisfying Case B.

In $b\left(\overline{C_{k}}\right)$, if the vertices in the red tree satisfy that each of them has blue edges connecting to $R_{1 b}$, or green edges connecting to $R_{1 g}$, then all the vertices in $b\left(\overline{C_{k}}\right)$ can be connected into the blue tree of $K\left(R_{1 b} \cup C_{k}, R_{2 b} \cup D_{k}\right)$ or the green tree of $K\left(R_{1 g} \cup\right.$ $\left.\overline{C_{k}}, R_{2 g} \cup \overline{D_{k}}\right)$ by the vertices in $b\left(D_{k}\right), R_{1 b}$ and $R_{1 g}$. Thus, the vertices of $K(A, B)$ can be covered by a blue tree, a green tree and at most one red tree. For $b\left(\overline{D_{k}}\right)$, we have a similar property. We may assume therefore that there exist vertices $x \in b\left(\overline{C_{k}}\right)$ and $y \in b\left(\overline{D_{k}}\right)$ such that $x$ and $y$ are the vertices in the red trees, and $K\left(x, R_{1 b} \cup R_{1 g}\right), K\left(y, R_{2 b} \cup R_{2 g}\right)$ are red stars. By Remark 5, we can suppose that $u v$ is a red edge in $K\left(R_{1 b}^{(1)}, R_{2 b}^{(1)}\right)$, then the red edges $u x, v y$ and $u v$ can connect the two red trees of $K\left(b\left(D_{k}\right), b\left(\overline{C_{k}}\right)\right)$ and $K\left(b\left(C_{k}\right), b\left(\overline{D_{k}}\right)\right)$ into one red tree. By Remark $6, K\left(R_{1 b}^{(1)}-u, R_{2 b}^{(1)}-v\right)$ has at least one blue edge. So, $K\left(\left(R_{1 b}-u\right) \cup C_{k},\left(R_{2 b}-v\right) \cup D_{k}\right)$ still has a blue spanning tree. Thus, the vertices of $K(A, B)$ can be covered by a blue tree, a green tree and a red tree.

Subcase 7.4.2. For any partitions $C=C_{i} \cup \overline{C_{i}}$ and $D=D_{j} \cup \overline{D_{j}}$, either $\left|b\left(C_{i}\right)\right| \geq$ $\left|b\left(\overline{C_{i}}\right)\right|>\left|b\left(D_{j}\right)\right| \geq\left|b\left(\overline{D_{j}}\right)\right|$, or $\left|b\left(D_{j}\right)\right| \geq\left|b\left(\overline{D_{j}}\right)\right|>\left|b\left(C_{i}\right)\right| \geq\left|b\left(\overline{C_{i}}\right)\right|$.

Without loss of generality, suppose $C=C_{k} \cup \overline{C_{k}}, D=D_{k} \cup \overline{D_{k}}$ such that $\left|b\left(D_{k}\right)\right| \geq$ $\left|b\left(\overline{D_{k}}\right)\right|>\left|b\left(C_{k}\right)\right| \geq\left|b\left(\overline{C_{k}}\right)\right|$. Define $X_{b}\left(C_{k}\right)=\left\{x \in R_{22} \mid x u\right.$ is a blue edge for some $u \in C_{k}$, $x v$ is a green edge for some $\left.v \in \overline{C_{k}}\right\}, X_{b}\left(\overline{C_{k}}\right)=\left\{x \in R_{22} \mid x u\right.$ is a green edge for some $u \in C_{k}, x v$ is a blue edge for some $v \in \overline{C_{k}}$.

Clearly, $b\left(C_{k}\right) \subseteq X_{b}\left(C_{k}\right), b\left(\overline{C_{k}}\right) \subseteq X_{b}\left(\overline{C_{k}}\right)$ and $X_{b}\left(C_{k}\right) \cup X_{b}\left(\overline{C_{k}}\right)=R_{22}$. Then at least one of $\left|X_{b}\left(C_{k}\right)\right| \geq \frac{1}{2}\left|R_{22}\right|$ and $\left|X_{b}\left(\overline{C_{k}}\right)\right| \geq \frac{1}{2}\left|R_{22}\right|$ holds.

Subcase 7.4.2.1. $\left|X_{b}\left(C_{k}\right)\right| \geq \frac{1}{2}\left|R_{22}\right|$.

In Subcase 7.4.1, we mainly use the property of $b\left(C_{k}\right)$ that every vertex in $b\left(C_{k}\right)$ has blue edges to $C_{k}$ and has green edges to $\overline{C_{k}}$. $X_{b}\left(C_{k}\right)$ also has the property. So, we consider $K\left(b\left(D_{k}\right), b\left(\overline{C_{k}}\right)\right)$ and $K\left(b\left(\overline{D_{k}}\right), X_{b}\left(C_{k}\right)\right)$ by the same argument as in Subcase 7.4.1. If $\left|X_{b}\left(C_{k}\right)\right| \geq\left|b\left(\overline{D_{k}}\right)\right|$, then the vertices of $K(A, B)$ can be covered by three vertex-disjoint monochromatic trees just as Subcase 7.4.1. Hence, we consider the case $\left|X_{b}\left(C_{k}\right)\right|<\left|b\left(\overline{D_{k}}\right)\right|$. In this case, $b\left(\overline{C_{k}}\right)$ and $b\left(\overline{D_{k}}\right)$ correspond to the partite set $A$ in Lemma 6 . Then by Lemma $6, K\left(b\left(D_{k}\right), b\left(\overline{C_{k}}\right)\right)$ has a tree partition satisfying Case A or Case $\mathrm{B}$, and $K\left(b\left(\overline{D_{k}}\right), X_{b}\left(C_{k}\right)\right)$ has a tree partition satisfying Case A, Case B or Case C. If $K\left(b\left(\overline{D_{k}}\right), X_{b}\left(C_{k}\right)\right)$ has a tree partition satisfying Case $\mathrm{A}$ or Case $\mathrm{B}$, then the proof is similar to that of Subcase 7.4.1. If $K\left(b\left(\overline{D_{k}}\right), X_{b}\left(C_{k}\right)\right)$ always has a tree partition satisfying Case $\mathrm{C}$, then denote the set of isolated vertices in Case $\mathrm{C}$ as $I\left(\overline{D_{k}}\right)$. We choose a tree partition of $K\left(b\left(\overline{D_{k}}\right), X_{b}\left(C_{k}\right)\right)$ such that $I\left(\overline{D_{k}}\right)$ is as small as possible. Clearly, 
$\left|b\left(\overline{D_{k}}\right)-I\left(\overline{D_{k}}\right)\right| \geq\left|X_{b}\left(C_{k}\right)\right|$. If every vertex in $I\left(\overline{D_{k}}\right)$ has blue edges to $R_{2 b}$ or has green edges to $R_{2 g}$, then similar to Subcase 7.4.1, the vertices of $K(A, B)$ can be covered by at most three vertex-disjoint monochromatic trees.

In the following, we assume that $I\left(\overline{D_{k}}\right)$ has at least one vertex such that all the edges incident with it in $K\left(I\left(\overline{D_{k}}\right), R_{2 b} \cup R_{2 g}\right)$ are red. Without loss of generality, suppose $\left|R_{2 b}^{(1)}\right| \geq\left|R_{2 g}^{(1)}\right|$, then we consider $K\left(I\left(\overline{D_{k}}\right), R_{2 b}^{(1)}\right)$. Clearly, all the edges of $K\left(I\left(\overline{D_{k}}\right), R_{2 b}^{(1)}\right)$ are blue or red.

Claim 3. If $\left|I\left(\overline{D_{k}}\right)\right| \leq\left|R_{2 b}^{(1)}\right|$, then the vertices of $K(A, B)$ can be covered by at most three vertex-disjoint monochromatic trees.

Proof. In $K\left(I\left(\overline{D_{k}}\right), R_{2 b}^{(1)}\right)$, since $\left|I\left(\overline{D_{k}}\right)\right| \leq\left|R_{2 b}^{(1)}\right|$, it is easy to see that we have the fact that some vertices in $I\left(\overline{D_{k}}\right)$ are in blue trees and the others are in a red star. If $K\left(b\left(D_{k}\right), b\left(\overline{C_{k}}\right)\right)$ has a tree partition satisfying Case A, or Case B such that in $b\left(\overline{C_{k}}\right)$ all the vertices of the red tree have green edges to $R_{1 g}$ or blue edges to $R_{1 b}$, then the vertices of $K(A, B)$ can be covered by a blue tree, a green tree and a red star. Otherwise, $K\left(b\left(D_{k}\right), b\left(\overline{C_{k}}\right)\right)$ always has a tree partition satisfying Case $\mathrm{B}$, and in $b\left(\overline{C_{k}}\right)$ there exists at least one vertex of the red tree such that all the edges incident with it in $K\left(b\left(\overline{C_{k}}\right), R_{1 g}\right)$ are red. Then, similar to Subcase 7.4.1.3, we can find a red edge $u v$ in $K\left(R_{1 g}^{(1)}, R_{2 b}^{(1)}\right)$, and it can connect these two red trees into one red tree, since $K\left(R_{1 g}^{(1)}, R_{2 b}^{(1)}\right)$ is a red complete bipartite graph. Thus, the vertices of $K(A, B)$ can be covered by a blue tree, a green tree and a red tree.

If $\left|I\left(\overline{D_{k}}\right)\right|>\left|R_{2 b}^{(1)}\right|$, then $\left|b\left(D_{k}\right)\right| \geq\left|b\left(\overline{D_{k}}\right)\right| \geq\left|I\left(\overline{D_{k}}\right)\right|+\left|X_{b}\left(C_{k}\right)\right|>\left|R_{2 b}^{(1)}\right|+\frac{1}{2}\left|R_{22}\right|$, and so $\left|b\left(D_{k}\right)\right|+\left|b\left(\overline{D_{k}}\right)\right|>\left|R_{2 b}^{(1)}\right|+\left|R_{2 g}^{(1)}\right|+\left|R_{22}\right|$. Thus, in Subcase 7.4.2.1, except $\left|b\left(D_{k}\right)\right|+$ $\left|b\left(\overline{D_{k}}\right)\right|>\left|R_{2 b}^{(1)}\right|+\left|R_{2 g}^{(1)}\right|+\left|R_{22}\right|$, the vertices of $K(A, B)$ can be covered by at most three vertex-disjoint monochromatic trees.

Subcase 7.4.2.2. $\left|X_{b}\left(\overline{C_{k}}\right)\right| \geq \frac{1}{2}\left|R_{22}\right|$.

In this case, we consider $K\left(b\left(D_{k}\right), b\left(C_{k}\right)\right)$ and $K\left(b\left(\overline{D_{k}}\right), X_{b}\left(\overline{C_{k}}\right)\right)$. Since $K\left(R_{1 b}^{(1)}, R_{2 b}^{(1)}\right)$ has at least one blue edge, $K\left(R_{1 g}^{(1)}, R_{2 g}^{(1)}\right)$ has at least one green edge. We know that $K\left(R_{1 b} \cup \overline{C_{k}}, R_{2 b} \cup D_{k}\right)$ has a blue spanning tree, and $K\left(R_{1 g} \cup C_{k}, R_{2 g} \cup \overline{D_{k}}\right)$ has a green spanning tree. We hope that the vertices of $b\left(\overline{D_{k}}\right)$ and $b\left(C_{k}\right)$ can be connected to the blue tree of $K\left(R_{1 b} \cup \overline{C_{k}}, R_{2 b} \cup D_{k}\right)$ and the green tree of $K\left(R_{1 g} \cup C_{k}, R_{2 g} \cup \overline{D_{k}}\right)$, or they can constitute a red tree. In this case, $b\left(C_{k}\right)$ and $b\left(\overline{D_{k}}\right)$ correspond to the partite set $A$ in Lemma 6. Similar to Subcase 7.4.2.1, we can get the fact that except $\left|b\left(D_{k}\right)\right|+\left|b\left(\overline{D_{k}}\right)\right|>$ $\left|R_{2 b}^{(1)}\right|+\left|R_{2 g}^{(1)}\right|+\left|R_{22}\right|$, the vertices of $K(A, B)$ can be covered by at most three vertexdisjoint monochromatic trees.

By Subcase 7.4.2.1 and Subcase 7.4.2.2, we have that for partitions $C=C_{k} \cup \overline{C_{k}}$ and $D=D_{k} \cup \overline{D_{k}}$ such that $\left|b\left(D_{k}\right)\right| \geq\left|b\left(\overline{D_{k}}\right)\right|>\left|b\left(C_{k}\right)\right| \geq\left|b\left(\overline{C_{k}}\right)\right|$, except $\left|b\left(D_{k}\right)\right|+\left|b\left(\overline{D_{k}}\right)\right|>$ $\left|R_{2 b}^{(1)}\right|+\left|R_{2 g}^{(1)}\right|+\left|R_{22}\right|$, the vertices of $K(A, B)$ can be covered by at most three vertex-disjoint monochromatic trees. If there exists a partition $D=D_{l} \cup \overline{D_{l}}$ such that $\left|b\left(C_{k}\right)\right| \geq\left|b\left(\overline{C_{k}}\right)\right|>$ $\left|b\left(D_{l}\right)\right| \geq\left|b\left(\overline{D_{l}}\right)\right|$, then by a similar argument to the above, we can obtain that except $\left|b\left(C_{k}\right)\right|+\left|b\left(\overline{C_{k}}\right)\right|>\left|R_{1 b}^{(1)}\right|+\left|R_{1 g}^{(1)}\right|+\left|R_{12}\right|$, the vertices of $K(A, B)$ can be covered by at most 
three vertex-disjoint monochromatic trees. But $\left|b\left(C_{k}\right)\right|+\left|b\left(\overline{C_{k}}\right)\right|>\left|R_{1 b}^{(1)}\right|+\left|R_{1 g}^{(1)}\right|+\left|R_{12}\right|$ contradicts to $\left|b\left(C_{k}\right)\right|+\left|b\left(\overline{C_{k}}\right)\right|<\left|b\left(D_{k}\right)\right|+\left|b\left(\overline{D_{k}}\right)\right|<\left|R_{12}\right|$. Thus, in the following we consider the case that for any partition $D=D_{i} \cup \overline{D_{i}}$, we always have $\left|b\left(D_{i}\right)\right| \geq\left|b\left(\overline{D_{i}}\right)\right|>$ $\left|b\left(C_{k}\right)\right| \geq\left|b\left(\overline{C_{k}}\right)\right|$, and $\left|b\left(D_{i}\right)\right|+\left|b\left(\overline{D_{i}}\right)\right|>\left|R_{2 b}^{(1)}\right|+\left|R_{2 g}^{(1)}\right|+\left|R_{22}\right|$, otherwise, the vertices of $K(A, B)$ can be covered by at most three vertex-disjoint monochromatic trees. Since $|D| \geq 3$, we have $\left|R_{12}\right|=\sum_{D=D_{i} \cup \overline{D_{i}}}\left|b\left(D_{i}\right) \cup b\left(\overline{D_{i}}\right)\right|=\sum_{D=D_{i} \cup \overline{D_{i}}}\left[\left|b\left(D_{i}\right)\right|+\left|b\left(\overline{D_{i}}\right)\right|\right]$

$$
>2\left(\left|R_{2 b}^{(1)}\right|+\left|R_{2 g}^{(1)}\right|+\left|R_{22}\right|\right)+\left|b\left(D_{k}\right)\right|+\left|b\left(\overline{D_{k}}\right)\right| \text {. }
$$

By Remark 3, $\left|R_{1 b}^{(1)}\right|+\left|R_{2 b}^{(1)}\right| \geq|C|+|D|$, that is, $\left|R_{1 b}^{(1)}\right|-|D| \geq|C|-\left|R_{2 b}^{(1)}\right|$.

$\left|R_{2}\right|+|D|=\left|R_{1}\right|+|C|>|C|+\left|R_{1 b}\right|+\left|R_{1 g}\right|+2\left(\left|R_{2 b}^{(1)}\right|+\left|R_{2 g}^{(1)}\right|+\left|R_{22}\right|\right)+\left|b\left(D_{k}\right)\right|+\left|b\left(\overline{D_{k}}\right)\right|$. $\left|R_{2 b}^{(2)}\right|+\left|R_{2 g}^{(2)}\right|>-|D|-\left|R_{22}\right|-\left|R_{2 b}^{(1)}\right|-\left|R_{2 g}^{(1)}\right|+|C|+\left|R_{1 b}\right|+\left|R_{1 g}\right|$

$+2\left(\left|R_{2 b}^{(1)}\right|+\left|R_{2 g}^{(1)}\right|+\left|R_{22}\right|\right)+\left|b\left(D_{k}\right)\right|+\left|b\left(\overline{D_{k}}\right)\right|$

$\geq 2|C|-2\left|R_{2 b}^{(1)}\right|-\left|R_{22}\right|-\left|R_{2 g}^{(1)}\right|+\left|R_{1 b}^{(2)}\right|+\left|R_{1 g}\right|$

$+2\left(\left|R_{2 b}^{(1)}\right|+\left|R_{2 g}^{(1)}\right|+\left|R_{22}\right|\right)+\left|b\left(D_{k}\right)\right|+\left|b\left(\overline{D_{k}}\right)\right|$

$>\left|b\left(D_{k}\right)\right|+\left|b\left(\overline{D_{k}}\right)\right|$.

Since $\left|b\left(D_{k}\right)\right| \geq\left|b\left(\overline{D_{k}}\right)\right|$, at least one of $\left|R_{2 b}^{(2)}\right|>\left|b\left(\overline{D_{k}}\right)\right|$ and $\left|R_{2 g}^{(2)}\right|>\left|b\left(\overline{D_{k}}\right)\right|$ holds. Without loss of generality, we assume $\left|R_{2 b}^{(2)}\right|>\left|b\left(\overline{D_{k}}\right)\right|$.

In the following, we consider $K\left(b\left(\overline{D_{k}}\right), R_{2 b}^{(2)}\right)$ and $K\left(b\left(D_{k}\right), b\left(\overline{C_{k}}\right)\right) . b\left(\overline{C_{k}}\right)$ and $b\left(\overline{D_{k}}\right)$ correspond to the partite set $A$ in Lemma 6 . Clearly, $K\left(b\left(D_{k}\right), b\left(\overline{C_{k}}\right)\right)$ has a tree partition satisfying Case A or Case B. Let $X=\left\{v \in b\left(\overline{D_{k}}\right) \mid K\left(v, R_{2 b}^{(2)}\right)\right.$ has blue edge $\}$, and $Y$ be the minimum subset of $R_{2 b}^{(2)}$ satisfying that for any $v \in X$, there exists a vertex $u \in Y$ such that $u v$ is a blue edge. Clearly, $|Y| \leq|X|$. Denote $P=b\left(\overline{D_{k}}\right)-X$ and $Q=R_{2 b}^{(2)}-Y$. Then all the edges of $K(P, Q)$ are red or green, and $|P|<|Q|$. We consider the following five small cases.

(1) $K(P, Q)$ has a green spanning tree.

If $K\left(P, R_{2 g}^{(1)}\right)$ has at least one green edge, then all the vertices in $P$ and $Q$ can be connected to the green tree of $K\left(R_{1 g} \cup \overline{C_{k}}, R_{2 g} \cup \overline{D_{k}}\right)$. So, the vertices of $K(A, B)$ can be covered by at most three vertex-disjoint monochromatic trees. We may assume therefore that $K\left(P, R_{2 g}^{(1)}\right)$ is a red complete bipartite graph. Let $u v$ be a red edge in $K\left(R_{1 g}^{(1)}, R_{2 g}^{(1)}\right)$, then $K(P, v)$ is a red star. Similarly, we can obtain that the vertices of $K(A, B)$ can be covered by three vertex-disjoint monochromatic trees.

(2) $K(P, Q)$ has a red spanning tree.

If there exists a vertex $x \in P$ such that $K\left(x, R_{2 g}^{(1)}\right)$ is a red star, let $u v$ be a red edge in $K\left(R_{1 g}^{(1)}, R_{2 g}^{(1)}\right)$, then $K(P, Q \cup v)$ has a red spanning tree. Similarly, the vertices of $K(A, B)$ can be covered by three vertex-disjoint monochromatic trees. Otherwise, every vertex in $P$ has green edge to $R_{2 g}^{(1)}$, then it is easy to prove that the vertices of $K(A, B)$ can be covered by at most three vertex-disjoint monochromatic trees.

(3) $K(P, Q) \in \mathcal{M}$.

Since $K(P, Q) \in \mathcal{M}$, we can give the partitions $P=P_{1} \cup P_{2}$ and $Q=Q_{1} \cup Q_{2}$ such 
that $K\left(P_{1}, Q_{1}\right)$ and $K\left(P_{2}, Q_{2}\right)$ are green complete bipartite graphs, and $K\left(P_{1}, Q_{2}\right)$ and $K\left(P_{2}, Q_{1}\right)$ are red complete bipartite graphs.

If both $K\left(P_{1}, R_{2 g}^{(1)}\right)$ and $K\left(P_{2}, R_{2 g}^{(1)}\right)$ have green edges, then it is easy to prove that the vertices of $K(A, B)$ can be covered by at most three vertex-disjoint monochromatic trees. If both $K\left(P_{1}, R_{2 g}^{(1)}\right)$ and $K\left(P_{2}, R_{2 g}^{(1)}\right)$ do not have green edges, then $K\left(P_{1} \cup P_{2}, R_{2 g}^{(1)}\right)$ is a red complete bipartite graph. Similarly, the vertices of $K(A, B)$ can be covered by three vertex-disjoint monochromatic trees. Without loss of generality, we may assume therefore that $K\left(P_{1}, R_{2 g}^{(1)}\right)$ has a green edge, say $w u$, and $K\left(P_{2}, R_{2 g}^{(1)}\right)$ is a red complete bipartite graph, then $K\left(P_{2}, R_{2 g}^{(1)}-u\right)$ is also a red complete bipartite graph. Clearly, the vertices of $K(A, B)$ can be covered by three vertex-disjoint monochromatic trees.

(4) $K(P, Q) \in \mathcal{S}_{1}$.

Since $|P|<|Q|, K(P, Q)$ has a green tree containing all the vertices in $P$. Then the proof is similar to the case that $K(P, Q)$ has a green spanning tree.

(5) $K(P, Q) \in \mathcal{S}_{2}$.

Since $K(P, Q) \in \mathcal{S}_{2}$, we can give partitions $P=P_{r} \cup P_{g}$ and $Q=Q_{r} \cup Q_{g}$ such that $K\left(P_{r}, Q_{r}\right)$ has a red spanning tree, $K\left(P_{g}, Q_{g}\right)$ has a green spanning tree. We consider four small subcases.

- At least one vertex in $P_{g}$ has green edge to $R_{2 g}^{(1)}$, and every vertex in $P_{r}$ has green edge to $R_{2 g}^{(1)}$.

- At least one vertex in $P_{g}$ that is incident with a green edge to $R_{2 g}^{(1)}$, and at least one vertex in $P_{r}$ such that all the edges incident with it are red in $K\left(P_{r}, R_{2 g}^{(1)}\right)$.

edge.

- $K\left(P_{g}, R_{2 g}^{(1)}\right)$ is a red complete bipartite graph, and $K\left(P_{r}, R_{2 g}^{(1)}\right)$ has at least one red

- $K\left(P_{g}, R_{2 g}^{(1)}\right)$ is a red complete bipartite graph, and $K\left(P_{r}, R_{2 g}^{(1)}\right)$ is a green complete bipartite graph.

For each of the above small subcases, we can easily obtain that the vertices of $K(A, B)$ can be covered by two or three vertex-disjoint monochromatic trees.

For Case 8, without loss of generality, suppose $K\left(R_{1}, D\right) \in \mathcal{S}_{1}, K\left(R_{2}, C\right) \in \mathcal{S}_{2}$. Since $K\left(R_{1}, D\right) \in \mathcal{S}_{1}$, we can denote $R_{1}=R_{1 b} \cup R_{12} \cup R_{1 g}$ or $D=D_{b} \cup D_{2} \cup D_{g}$. Similarly, we can denote $R_{2}=R_{2 b} \cup R_{22} \cup R_{2 g}$ or $C=C_{b} \cup C_{2} \cup C_{g}$. The case $R_{1}=R_{1 b} \cup R_{12} \cup R_{1 g}$ and $C=C_{b} \cup C_{2} \cup C_{g}$, and the case $R_{2}=R_{2 b} \cup R_{22} \cup R_{2 g}$ and $D=D_{b} \cup D_{2} \cup D_{g}$ are similar to Subcase 6.1 and Subcase 6.2, respectively. The case $C=C_{b} \cup C_{2} \cup C_{g}$ and $D=D_{b} \cup D_{2} \cup D_{g}$ is similar to Subcase 7.3. In the following, we consider the case $R_{1}=R_{1 b} \cup R_{12} \cup R_{1 g}$ and $R_{2}=R_{2 b} \cup R_{22} \cup R_{2 g}$. The proof is similar to that of Subcase 7.4, by considering two subcases:

Subcase 8.1. There exist partitions $C=C_{k} \cup \overline{C_{k}}$ and $D=D_{k} \cup \overline{D_{k}}$ such that $\left|b\left(C_{k}\right)\right| \geq$ $\left|b\left(\overline{D_{k}}\right)\right|$ and $\left|b\left(D_{k}\right)\right| \geq\left|b\left(\overline{C_{k}}\right)\right|$.

This case can be proved similarly to Subcase 7.4.1. 
Subcase 8.2. For any partitions $C=C_{i} \cup \overline{C_{i}}$ and $D=D_{j} \cup \overline{D_{j}}$, either $\left|b\left(C_{i}\right)\right| \geq\left|b\left(\overline{C_{i}}\right)\right|>$ $\left|b\left(D_{j}\right)\right| \geq\left|b\left(\overline{D_{j}}\right)\right|$, or $\left|b\left(D_{j}\right)\right| \geq\left|b\left(\overline{D_{j}}\right)\right|>\left|b\left(C_{i}\right)\right| \geq\left|b\left(\overline{C_{i}}\right)\right|$.

Since $K\left(R_{2}, C\right) \in \mathcal{S}_{2}$, either $\min \left\{\left|R_{2 b}\right|,\left|R_{2 g}\right|\right\}=1$, or there exists a partition $C=$ $C_{i} \cup \overline{C_{i}}$ such that $b\left(\overline{C_{i}}\right)=\emptyset$. If $\min \left\{\left|R_{2 b}\right|,\left|R_{2 g}\right|\right\}=1$, then the result is obvious. We may assume therefore that $R_{22}=\cup_{C=C_{i} \cup \overline{C_{i}}}\left[b\left(C_{i}\right) \cup b\left(\overline{C_{i}}\right)\right]$ such that for some $\overline{C_{i}}, b\left(\overline{C_{i}}\right)=\emptyset$. Without loss of generality, suppose $b\left(\overline{C_{l}}\right)=\emptyset$, then we consider partitions $C=C_{l} \cup \overline{C_{l}}$ and $D=D_{j} \cup \overline{D_{j}}$ for some $j$, hence $\left|b\left(D_{j}\right)\right| \geq\left|b\left(\overline{D_{j}}\right)\right|>\left|b\left(C_{l}\right)\right| \geq\left|b\left(\overline{C_{l}}\right)\right|$. Thus, we can prove it similarly to Subcase 7.4.2.

Up to now, we have exhausted all cases, and proved that for any 3-edge-colored complete bipartite graph $K(n, n)$ satisfying the condition of Theorem 7 , the vertices of it can be covered by at most three vertex-disjoint monochromatic trees. Thus $t_{3}(k(n, n)) \leq 3 . \square$

\section{Conclusion}

As one can see, we only considered 3-edge-colored complete bipartite graphs with "equal bipartition", and the "color degree" of every vertex is 3. These restrictions are really very helpful to concluding our proofs. Even though, the proof looks very long and complicated. More general questions are: can we drop the equal bipartition restriction to get the partition number? can we drop the color degree restriction to get the partition number? or can we drop both restrictions to get the partition number? We tried for a year but failed to complete it. Things become out of control without any of the restrictions.

Acknowledgement: The authors are very grateful to the referees for their comments and suggestions which helped to improve the presentation of the original manuscript.

\section{References}

[1] P. Erdős, A. Gyárfás and L. Pyber, Vertex coverings by monochromatic cycles and trees, J. Combin. Theory, Ser. B 51 (1991), 90-95.

[2] A. Hajnal, P. Komjáth, L. Soukup and I. Szalkai, Decompositions of edge colored infinite complete graphs, Colloq. Math. Soc. János Bolyai 52 (1987), 277-280.

[3] P.E. Haxell and Y. Kohayakawa, Partitioning by monochromatic trees, J. Combin. Theory. Ser. B 68 (1996), 218-222.

[4] Z.M. Jin, M. Kano, X. Li and B. Wei, Partitioning 2-edge-colored complete multipartite graphs into monochromatic cycles, paths and trees, J. Comb. Optim 11 (2006), 445-454.

[5] A. Kaneko, M. Kano and K. Suzuki, Partitioning complete multipartite graphs by monochromatic trees, J. Graph Theory 48 (2005), 133-141.

[6] M. Kano and X. Li, Monochromatic and heterochromatic subgraphs in edge-colored graphs - a survey, Graphs and Combin. 24(4)(2008), 237-263. 